\title{
Perfil de Tabagistas da área de abrangência de uma Estratégia de Saúde da Família
}

\author{
Profile of Smokers in the area covered by a Family Health Strategy
}

Priscilla Rodrigues Caminha Carneiro ${ }^{1}$, Adriana Paiva Camargo Saraiva ${ }^{2}$, Deliane de Lucia Pereira Silva ${ }^{3}$, Terezinha Corrêa Cunha ${ }^{3}$

\section{RESUMO}

Objetivou-se identificar o perfil dos tabagistas adultos e usuários de uma estratégia saúde da família do Sul do Pará. Trata-se de um estudo transversal de abordagem quantitativa, descritiva e analítica, a amostra compreendeu a população adulta de 20 a 59 anos, foi constituída por 22 tabagistas, $(n=12 ; 55 \%)$ eram do sexo feminino, $(n=10$; $45 \%$ ) sexo masculino, (82\%) cor parda, (77\%) estado civil solteiro, (68\%) desempregado e $(82 \%)$ com baixo grau de escolaridade, $86 \%$ dos participantes faziam consumo do cigarro associado ao álcool, e o grau de dependência a nicotina elevado (36\%). O conhecimento do perfil dos tabagistas, possibilita a equipe da unidade de saúde melhorar as estratégias antitabágicas e a adesão dos fumantes ao programa de abordagem cognitiva e comportamental e o tratamento medicamentoso, pois apesar das 12 Estratégias Saúde da Família existentes no município apenas a referida unidade estudada desenvolve o programa, no entanto com taxa de adesão.

Palavras-chave: Cessação; Dependência; Doença Crônica; Prevalência; Tabaco.
${ }^{1}$ Enfermeira. Especialista em Saúde Pública com ênfase em Saúde da Familia. Docente do Departamento de Enfermagem Hospitalar da Universidade do Estado do Pará.

E-mail:

priscillarcaminha@hotmail.com

²Odontóloga. Enfermagem Fundamental. Departamento de Morfologia e Ciências Fisiológicas da Universidade do Estado do Pará.

Enfermeira, Universidade do Estado do Pará.

\section{ABSTRACT}

The aim of this study was to identify the profile of adult smokers and users of a family health strategy in Southern Pará. This was a cross-sectional study with a quantitative, descriptive and analytical approach, the sample comprised the adult population aged 20 to 59 years. $(n=12,55 \%)$ were female, $(n=10 ; 45 \%)$ male, $(82 \%)$ brown, $(77 \%)$ unmarried, $68 \%$ unemployed, and (82\%) with low educational level, $86 \%$ of the participants were using alcohol-related cigarette, and the high degree of addiction to nicotine (36\%). The knowledge of the smokers' profile allows the health unit team to improve the anti-smoking strategies and the adherence of smokers to the program of cognitive and behavioral approach and drug treatment, because despite the 12 Family Health Strategies in the municipality, studied program, however with membership rate.

Keywords: Cessation; Dependency; Chronic disease; Prevalence; Tobacco. 


\section{INTRODUÇÁO}

O consumo de cigarro vem se tornando altamente elevado entre a população, tornando- se um problema de saúde pública, uma vez que seu uso contínuo contribui para o desenvolvimento de vários agravos na saúde, sendo considerado como a maior causa evitável de morte no Brasil e no mundo (BRASIL, 2015).

Estima-se que, um terço da população mundial adulta (1 bilhão e 200 milhões de pessoas), sejam fumantes (BRASIL, 2017). O cigarro pode causar várias consequências na vida do indivíduo, além de provocar sérios danos à saúde como câncer, doença cardiovascular, pulmonares, úlcera péptica entre outras patologias.

Aproximadamente 4.720 substâncias químicas estão contidas na fumaça dos derivados do tabaco, tornando o tabagismo o principal responsável por quase 50 doenças (BRASIL, 2017).

Além disso, o tabagismo é uma das maiores causas de Doenças Crônicas não Transmissíveis (DCNTs). Em 2007, cerca de 72\% das mortes no Brasil foram atribuídas às DCNTs como (doenças cardiovasculares, doenças respiratórias crônicas, diabetes, câncer e outras), e 10\% às doenças infecciosas e parasitárias e $5 \%$ aos distúrbios de saúde materno-infantis (NUNES, 2011).

Atualmente cinco milhões de pessoas morrem anualmente em decorrência das doenças que estão relacionadas ao cigarro, sendo que $80 \%$ delas ocorrem em países de baixa e média renda, e se a tendência atual continuar a estimativa é que em 2030 venha ocorrer cerca de oito milhões de mortes ao ano (BRASIL, 2001).

Portanto, é imprescindível discutir a tal temática, sabendo que no município onde a pesquisa foi realizada existem 12 Estratégias de Saúde da Família (ESF) e atualmente, apenas uma (1) desenvolve o Programa Nacional de Controle ao Tabagismo (PNCT), mas que no entanto há uma baixa adesão ao programa em relação ao número de fumantes existentes na área de abrangência da estratégia.

Para que haja uma maior adesão ao tratamento do tabagismo pelo SUS (abordagem cognitiva, comportamental e medicamentosa), é essencial que a equipe multidisciplinar reconheça as características gerais dos tabagistas de sua área de abrangência, para uma maior aproximação com público alvo e efetivação do programa de forma que atenda às necessidades específicas dessa população, desenvolvendo 
estratégias adequadas, para assim manter o controle e promover a cessação do tabagismo (CARAM, 2009).

Diante do exposto, o presente estudo propôs identificar o perfil dos fumantes adultos em usuários de uma Estratégia Saúde da Família (ESF) na zona urbana do Sul do Pará.

\section{MATERIAIS E METODOS}

Trata-se de um estudo transversal, de abordagem quantitativa descritiva e analítica, envolvendo uma amostra representativa da população adulta (20 a 59 anos). O estudo foi realizado em uma Estratégia Saúde da Família (ESF), do Sul do Pará.

A seleção da amostra foi realizada pelo método de amostragem por conglomerados. Participaram do estudo $(n=11)$ setores existentes nos 5 bairros da área de abrangência. De cada setor foi sorteado apenas um quarteirão e neste, uma esquina foi selecionada para o início do estudo, foram percorridos os seis primeiros domićlios, no sentido horário de cada esquina sorteada. O tamanho da amostra obtido foi de 66 famílias (números de setores 11×6 casas visitadas=66 famílias).

Foram incluídos na pesquisa todos os tabagistas, que pertencem à faixa de 20 a 59 anos de ambos os sexos, residentes dos bairros de abrangência da pesquisa e que aceitaram assinar o Termo de Consentimento Livre e Esclarecido (TCLE). Foram excluídos, indivíduos não tabagistas, menor de 20 e maior de 59 anos de idade de ambos os sexos, que não residem nos bairros de abrangência da pesquisa e não aceitaram participar da entrevista e assinar o TCLE.

Para a obtenção dos dados, foi aplicado um questionário contendo 20 questões contendo 14 questões que avaliam aspecto demográfico, socioeconômico e as condições e situações de saúde dos tabagistas, e para avaliar o grau de dependência da nicotina, foi aplicado o teste denominado Fagerström Test for Nicotine Dependence (FTND) validado para utilização no Brasil (ARAÚJO, 2007). Constitui-se em seis questões. A soma dos pontos resulta num escore, que varia de 0 a 10, e indica o grau de dependência do fumante em: muito baixo (0-2), baixo (3-4), médio (5), elevado (67) e muito elevado (8-10). 
Os dados foram tabulados no programa Microsoft Excel versão 2013 e foram analisados por meio de estatística descritiva em percentual para as variáveis.

A fim de garantir os princípios bioéticos dos participantes em relação à autonomia, não maleficência, beneficência, justiça e equidade, considerando que o tabagismo acarreta diversas consequências a saúde, os aspectos éticos da pesquisa visam atender às exigências éticas e científicas fundamentais definidas pela Resolução 466/2012 e outras normativas em vigor, que tratam de pesquisas envolvendo seres humanos, no âmbito do Conselho Nacional de Saúde Brasileiro, por meio da Comissão Nacional de ética em pesquisa (CONEP).

Portanto o estudo só teve início após a aprovação do Comitê de Ética em Pesquisa, mediante o parecer no 2.844.054 emitido pelo CEP no dia 25 de agosto de 2018. A coleta de dados ocorreu em setembro e outubro de 2018 e teve o consentimento escrito dos participantes, mediante a assinatura do TCLE.

\section{RESULTADOS}

De 66 famílias visitadas, 10 casas se encontraram fechadas, 19 famílias alegaram não possuir nenhum fumante, e apenas 37 famílias participaram da pesquisa. Foram entrevistadas 36 tabagistas, mas apenas 22 se enquadraram dentro dos critérios de inclusão, foram excluídos da amostra três tabagistas menores de 20 anos, oito com idade maior que 59 anos, três que se recusaram em assinar o TCLE.

$\mathrm{Na}$ tabela 1, estão a distribuição em números absolutos e percentual dos fumantes quantos as variáveis sociodemográficas.

De acordo com os resultados dos 22 participantes, mais da metade foram do sexo feminino com ( $n=12 ; 55 \%)$, e sexo masculino $(n=10 ; 45 \%)$. Houve um maior predomínio de tabagismo entre o sexo feminino. Verificou-se também, que a idade foi maior entre 50 a 59 anos, com ( $n=10 ; 45 \%)$ tabagistas, enquanto que, entre 20 a 29 anos foram cinco casos, entre 30 a 39 anos quatros tabagistas, e apenas três na faixa etária de 40 a 49 anos. Vale ressaltar que os dados apontaram maior número de fumante acima de 50 anos.

A análise de indicadores socioeconômicos descritas na (Tabela 1) apresenta uma diferença significante no que se refere a cor da pele, autodeclarados pardo $(n=18 ; 82 \%)$; preto dois $(9 \%)$ e branco dois (9\%). Quando questionado o grau de escolaridade dos entrevistados, os dados apontaram mais elevado com $(n=18 ; 82 \%)$ para o ensino 
fundamental incompleto, um (5\%) para o nível fundamental completo, um (5\%) para o nível médio, ensino superior zero ( $0 \%$ e os que nunca estudaram dois ( $9 \%)$. Isso demonstra que a população com menos grau de escolaridade, estão mais propicio a se tornarem fumantes.

Tabela 1-Características sociodemográficas dos tabagistas na referida área de abrangência. Conceição do Araguaia, PA, Brasil, 2018.

\begin{tabular}{lcc}
\hline CARACTERIZAÇÃO & N & $\%$ \\
\hline Tabagistas & 22 &
\end{tabular}

\section{Sexo}

Feminino

12

55

Masculino

$10 \quad 45$

Idade

20 a 29 anos

$5 \quad 23$

30 a 39 anos

$4 \quad 18$

40 a 49 anos

$3 \quad 14$

50 a 59 anos

10

45

Raça/cor

Branco

29

Preto

29

Parda

$18 \quad 82$

\section{Escolaridade}

Nível Fund. Incompleto $18 \quad 82$

Nível fundamental completo $\quad 1 \quad 5$

Ensino médio

$1 \quad 5$

Ensino superior

$0 \quad 0$

Nunca estudou

29

\section{Estado civil}

Solteira

$17 \quad 77$

Casada

$5 \quad 23$

Situação no mercado de trabalho

Empregado

Desempregado

29

Assalariado com carteira assinada Aposentado

$15 \quad 68$

$0 \quad 0$

15

Dos 22 entrevistados, $(n=17 ; 77 \%)$ são solteiros, e cinco (23\%) casados. Houve um percentual bem acentuado de tabagistas solteiras. 
Em relação a situação dos tabagistas no mercado de trabalho $(n=15 ; 68 \%)$ estão desempregados, dois (9\%) disseram ser empregados, e quatros (18\%) declararam-se autônomos. $O$ índice de desempregados foi bem perceptível na população adulta de 30 a 40 anos de idade.

As estatísticas descritivas sobre as condições e situações de saúde dos entrevistados são apresentadas a seguir na Tabela 2.

Tabela 2 - Condições e situações de saúde

\begin{tabular}{lcc}
\hline & Sim N (\%) & Não N (\%) \\
\hline Faz uso de álcool & $19(86)$ & $03(14)$ \\
Uso de outras drogas & $2(9)$ & $20(91)$ \\
Tem Hiper. Arterial & $05(23)$ & $17(77)$ \\
Tem Diabetes & $0(0)$ & $22(100)$ \\
Teve AVC & $0(0)$ & $22(100)$ \\
Teve Doença Cardiovascular & $2(9)$ & $20(91)$ \\
Tem ou teve Câncer & $0(100)$ & $22(100)$ \\
Doença resp. no pulmão & $6(27)$ & $16(73)$ \\
\hline
\end{tabular}

No que se refere a associação entre o uso do cigarro e consumo de bebida alcoólica, teve uma maior proporção com ( $n=19 ; 86 \%)$, em relação aos que consomem somente tabaco três (14\%), com maior prevalência entre a população adulta na faixa etária de 50 59 anos (8 casos). Enquanto ao uso de outras drogas ilícitas, dois (9\%) responderam fazer uso, e ( $n=20 ; 91 \%)$ consomem somente tabaco.

O número de tabagistas diagnosticado com Hipertensão Arterial Sistêmica (HAS), encontrada na amostra foi de cinco (23\%), sendo que a maioria ( $n=17,77 \%)$ são tabagistas normotensos.

Em relação, Acidente Vascular Encefálico- AVE, câncer e diabetes, todos os entrevistados $(n=22,100 \%)$ responderam nunca foram acometidos por essas patologias. Apenas dois (9\%) tabagistas relataram ser diagnosticados com doenças cardiovasculares, enquanto ( $n=20,91 \%)$ afirmaram não apresentar essa doença.

De acordo com os entrevistados a maioria $(n=16 ; 73 \%)$ não possui nenhum problema respiratório no pulmão, apenas seis (27\%) tabagistas responderam ter problemas pulmonares. 
A tabela 3, descreve o questionário de tolerância de Fagerström, sobre o grau de dependência a nicotina dos tabagistas.

Tabela 3 - Questionários de tolerância de Fagerström

\begin{tabular}{|c|c|c|}
\hline & $\operatorname{Sim} N(\%)$ & Não N (\%) \\
\hline \multicolumn{3}{|l|}{$\begin{array}{l}\text { Quanto tempo após ao acordar você fuma } \\
\text { o primeiro cigarro? }\end{array}$} \\
\hline Dentro de 5 minutos & $6(27)$ & \\
\hline Entre 6 a 30 minutos & $12(55)$ & \\
\hline Entre 31 a 60 minutos & $3(14)$ & \\
\hline Após 60 minutos & $1(4)$ & \\
\hline $\begin{array}{l}\text { Você acha difícil não fumar em lugares } \\
\text { proibidos como igrejas, bibliotecas e etc.? }\end{array}$ & $8(36)$ & $14(64)$ \\
\hline $\begin{array}{l}\text { Qual cigarro do dia que traz mais } \\
\text { satisfação, é o primeiro da manhã? }\end{array}$ & $17(77)$ & $5(23)$ \\
\hline \multicolumn{3}{|l|}{ Quantos cigarros por dia? } \\
\hline Menos de 10 & $11(50)$ & \\
\hline De 11 a 20 & $5(23)$ & \\
\hline De 21 a 30 & $4(18)$ & \\
\hline Mais de 31 & $2(9)$ & \\
\hline $\begin{array}{l}\text { Você fuma mais frequentemente pela } \\
\text { manhã? }\end{array}$ & $13(59)$ & $9(41)$ \\
\hline $\begin{array}{l}\text { Você fuma mesmo doente quando precisa } \\
\text { ficar de cama a maior parte do tempo? }\end{array}$ & $11(50)$ & $11(50)$ \\
\hline
\end{tabular}

A maioria dos participantes $(n=12 ; 55 \%)$ afirmaram que acendiam o primeiro cigarro entre 6 a 30 minutos após acordar, e seis (27\%) dentro de 5 minutos, e entre 31 a 60 minutos três (14\%) pessoas e após 60 minutos um (4\%).

Com relação ao tempo que os indivíduos fumam o primeiro cigarro do dia $(n=12 ; 55 \%)$ dos entrevistados responderam fumar entre 6 e 30 minutos ao acordar. Quando perguntado se achavam difícil não fumar em lugares proibidos como igrejas, bibliotecas, a maioria $(\mathrm{n}=14 ; 64 \%)$ responderam não ter essa dificuldade enquanto oito (36\%) relataram que sim. 
O primeiro cigarro consumido no dia apresentou-se como mais prazeroso que qualquer outro para a maioria $(n=17 ; 77 \%)$ dos entrevistados, e somente cinco $(23 \%)$ disseram não ser o primeiro do dia. O consumo médio diário de cigarros dos fumantes desse estudo foi metade $(n=11 ; 50 \%)$, fumam menos de 10 cigarros ao dia, a outra metade variou-se entre cinco (23\%) de 11-20 cigarros, quatro (18\%) 21-30 cigarros, e apenas dois (9\%) consomem mais de 31 cigarros ao dia.

Os entrevistados que relataram consumir cigarros frequentemente pela manhã foram ( $n=13 ; 59 \%)$, o restante nove (41\%) consomem mais ao longo do dia.

$\mathrm{Na}$ referida pesquisa a metade $(\mathrm{n}=11 ; 50 \%)$ dos tabagistas que não interrompem a prática tabágica mesmo quando está doente. Os dados da amostra apresentado no Gráfico 1, descreve a interpretação dos resultados do teste de Fagerström do grau de dependência a nicotina. Descrevendo as principais variáveis do teste, muito baixo; baixo; médio; elevado e muito elevado.

Gráfico 1- Grau de dependência a nicotina (Teste de Fagerström).

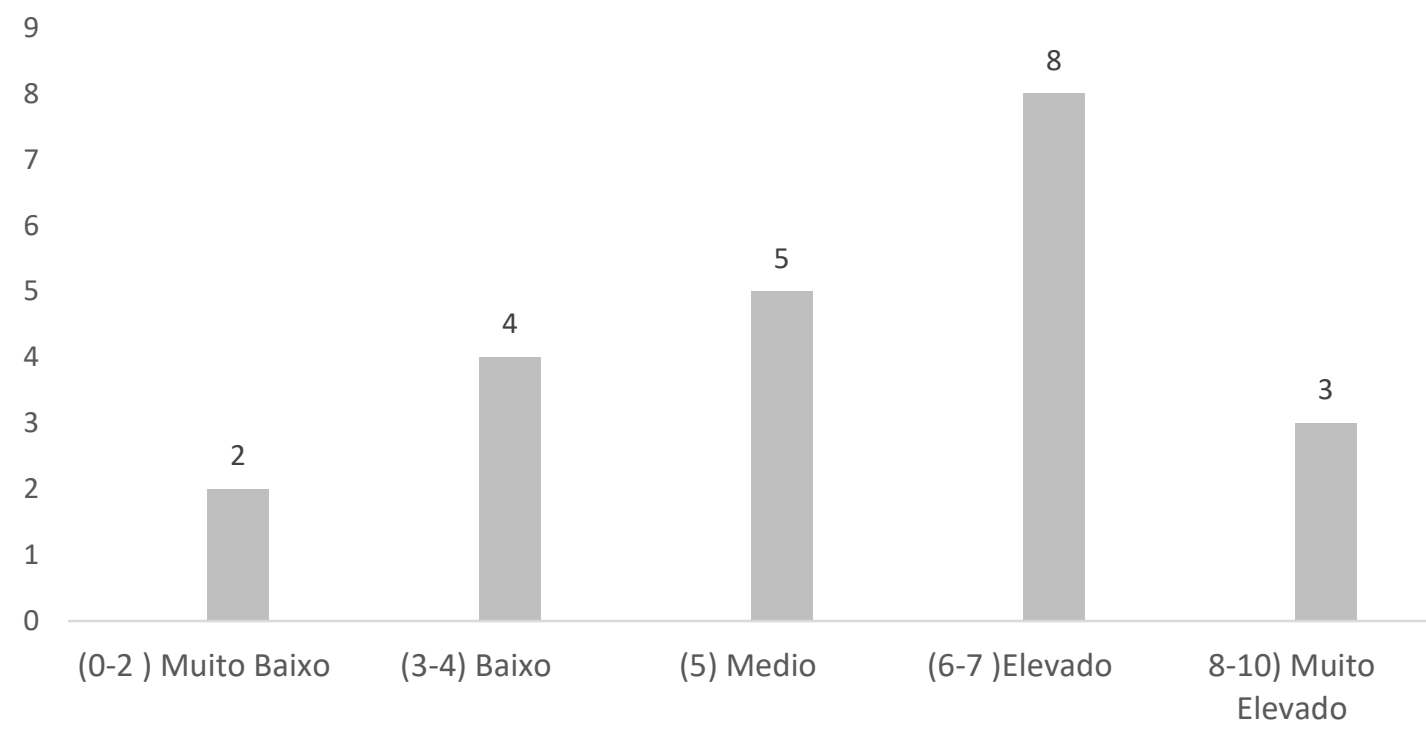

Em relação aos resultados do grau de dependência de nicotina houve uma prevalência de oito (36\%) no grau de dependência elevado, cinco (23\%) foram classificados como médio, quatro (18\%) como baixo, três (14\%) muito elevado e apenas dois (9\%) dos entrevistados apresentaram o menor grau de dependência a nicotina, muito baixo.

O presente estudo, identificou-se uma prevalência de tabagista no sexo feminino, sendo de acordo com a pesquisa de Caram (2009), que 63\% de seu resultado eram tabagistas feminino, e também com estudo de Zacan et al. (2011) com 76,6\% do sexo 
feminino e $23,4 \%$ do sexo masculino, o que demostra o nível elevado de fumo entre as mulheres, assim como estudo realizado em um centro de atendimento de saúde pública em Passo Fundo $76,6 \%$ eram do sexo feminino e $23,4 \%$ do sexo masculino, o que demostra 0 nível elevado de tabagismo entre as mulheres. Assim como um estudo realizado em Ponta Grossa, PR, onde houve predomínio das mulheres ( $n=63 ; 63 \%$ ) (MÜLLER, 2017).

Em relação a idade, houve um maior número de fumante acima de 50 anos. A prevalência do tabagismo em idosos verificada neste estudo (45\%) foi compatível com o estudo de Zaitune (2012) realizado em São Paulo, com 73\% de idosos tabagistas. Luppi (2008), vem afirmar que a dependência nicotínica aumenta progressivamente nas faixas etárias mais avançadas (entre 51 e 60 anos).

Houve um elevado percentual de tabagistas pardos indo de acordo com o esperado da pesquisa. Almeida e Mussi (2006) em Salvador, identificou também uma prevalência de tabagistas na cor parda de (45,5\%). Já em um estudo transversal realizado com a comunidade acadêmica de uma escola de enfermagem no sul do Brasil, em 2009 mostrou que a maioria dos participantes declararam ser de cor branca cerca de (85\%).Assim como a pesquisa realizada também no Sul do pais mais especificamente em Ponta Grossa - PR houve predomínio da cor branca ( $n=82$; 82\%) (MÜLLER, 2017). Justificam-se tais achados pelas marcas da colonização e da imigração que podem ser percebidas nas diferentes regiões do País, visto que a maior concentração de negros, $75 \%$, está nas regiões Norte e Nordeste. A Bahia, por exemplo, que recebeu grande fluxo de africanos, tem a maior população negra $(17,1 \%)$. No Sul e Sudeste, existem grupos de origem alemã, polonesa, ucraniana, italiana, japonesa e portuguesa, portanto essas regiões concentram a maior parte da população amarela e branca. Santa Catarina é o estado com maior número de pessoas que se autodeclaram brancas: 85,7\% (BRASIL, 2007).

Foi possível identificar que, quanto menor o grau de escolaridade, maior a prevalência de tabagismo, pois os dados apontaram mais elevado com $(n=18 ; 82 \%)$ para o ensino fundamental incompleto.

Dos entrevistados, $(n=17 ; 77 \%)$ são solteiros, resultado que vai contra os resultados de uma pesquisa realizada em Ponta Grossa, onde predominou o estado civil casado ( $n=49 ; 49 \%$ ) (MÜLLER, 2017).

Os resultados também apontam uma alta prevalência de tabagistas que faz uso do cigarro associado a bebida alcoólica. Na pesquisa de Almeida e Mussi (2006) o uso do cigarro foi mais frequente junto a bebida alcoólica $(36,4 \%)$ em Salvador. 
O número de Hipertensos identificados foi maior na faixa etária de 50-59 anos, dentre os 10 idosos (3 casos). Isso demonstra a população adulta com maior fator de risco para hipertensão, pois além do fumo, tabagismo, sedentarismo prevalece entre esses indivíduos.

Embora, os pacientes incluídos neste estudo, não apresentaram nenhuma patologia de pulmão, o Instituto Nacional de Câncer -INCA (2007), aponta o câncer de pulmão como a maior causa de mortalidade entre os tipos de câncer no Brasil, em 90\% dos casos diagnosticados, é associado ao consumo de tabaco. Estima-se que, todo ano mais de cinco milhões de pessoas morrem no mundo por causa do cigarro e $25 \%$ das mortes são por doenças do coração (BRASIL, 2017).

O tabagismo é um grave problema de Saúde Pública, é fator de risco para cerca de quase 50 diferentes doenças incapacitantes e fatais, dentre elas, doenças respiratórias, neoplasias malignas, doença pulmonar obstrutiva crônica-DPOC e doenças cardiovasculares, sendo o câncer de pulmão, a primeira causa de morte por câncer no Brasil, com $90 \%$ dos casos em fumantes (BRASIL, 2017).

Diante dos dados foi possível observar o auto índice de tabagistas que fumam após o café da manhã. Esse percentual elevado, vai de acordo com a pesquisa de Luppi (2008), de 111 tabagistas entrevistados, 93,3\% acendiam o primeiro cigarro do dia entre 30 minutos após despertar.

A dificuldade de fumar em ambiente fechado, está diretamente relacionado com o grau de dependência nicotina, esta variável para Halty et al. (2002) é importante critério para avaliação do grau de dependência. Na pesquisa de Luppi (2008), mostrou que primeiro cigarro do dia também foi o mais prazeroso.

Segundo Zaitune (2012), o número de cigarros fumados e a avaliação do tempo de fumar o primeiro cigarro após acordar também são indicadores da intensidade da dependência a nicotina. Essa dependência traz vários malefícios a saúde do tabagista. Rossaneis (2011) relata que, "o risco da hipertensão arterial associada ao tabagismo é proporcional ao número de cigarros fumados e à profundidade da inalação".

$\mathrm{Na}$ referida pesquisa a metade $11(50 \%)$ dos tabagistas que não interrompem a prática tabágica mesmo quando está doente, foi igual aos indivíduos que tem dificuldade de interromper 11 (50\%). Enquanto que, no estudo de Luppi (2006), 74,5\% dos indivíduos efetuaram uma ou mais tentativas em locais proibidos. Isso mostra o grau de dependência elevado da nicotina. 
Houve predomínio do grau de dependência a nicotina elevado, oito (36\%), esse elevado grau está de acordo com a pesquisa que avaliou a utilização do Questionário de Tolerância de Fagerström (QTF) como instrumento de medida da dependência nicotínica realizada em um Hospital Universitário é referência na cidade de Rio Grande, no Rio Grande do Sul, para pneumopatas crônicos, detectando 59 \% dos tabagistas com grau elevado de dependência a nicotina (HALTY, 2002).

O tabagista com grau de dependência elevado, tende a desencadear danos à saúde mais elevados, que em relação os de dependência leve. De acordo com o estudo do Dr. Rosenberg (2004), "o tratamento deve ser mais enérgico e mais prolongado que o geralmente recomendado. É indicado suporte psicológico, particularmente quando há estresse e alto consumo de álcool".

\section{CONSIDERAÇOEES FINAIS}

O presente estudo possibilitou identificar o perfil dos tabagistas adultos usuários de uma Estratégia de Saúde da Família, sendo predominante no sexo feminino, na cor parda, com idade entre 50 a 59 anos e com baixo grau de escolaridade. Além, do alto índice de tabagistas desempregados e solteiros.

Contudo, conclui-se que os perfis dos tabagistas estão de acordo com a hipótese da referida pesquisa, com exceção de sexo, que foi mais prevalecente no sexo feminino que no masculino.

Este estudo demostrou também que $86 \%$ dos participantes faziam consumo associado de álcool/tabaco. Sabendo que o tabagismo é fator de risco para as DCNTs e DPOC, com relação as patologias, houve um percentual menor do que o esperado, como hipertensão $23 \%$, e doenças cardíacas e pulmonares apenas $27 \%$.

Em relação ao grau de dependência a nicotina, foi encontrado um elevado grau de dependência nicotínica $36 \%$ seguido de $23 \%$ com dependência média. Os resultados demonstram que, a população pesquisada tem dificuldade de abandonar o fumo e aderir programa de cessação do tabagismo pelo alto grau de dependência, a condição social e o grau de escolaridade que podem influenciar na adesão ao tratamento, tanto em termos de compreensão do conteúdo, quanto na utilização adequada dos recursos necessários.

Segundo Pesquisa Nacional de Saúde realizada em 2013 a prevalência de fumantes no Pará é de 12,9\% entre adultos fumantes com 18 anos ou mais em amostras domiciliares 
(rural e urbana), cerca de 700.000 mil de pessoas e há cerca de 6.157 fumantes no município onde foi realizada a pesquisa. No entanto o Programa de Abordagem Cognitiva Comportamental e Medicamentosa que foi desenvolvido pelo Ministério da Saúde e o Instituto Nacional de Câncer - INCA que tem que como objetivo ajudar os participantes a deixarem de fumar, fornecendo-Ihes informações e estratégias necessárias para direcionar seus próprios esforços, ainda não alcançou sua cobertura máxima no município estudado, visto que das 12 ESF's existentes somente uma unidade desenvolve, ficando evidente que nem todo fumante possui o acesso adequado para iniciar seu tratamento.

Faz-se necessário preparar profissionais da saúde para lidar com habilidades multiprofissionais afim de efetivar tal programa com objetivo de promover o controle e a cessação do tabagismo no município.

Portanto, propõem-se parceria com as instituições de ensino em saúde para o combate dessa epidemia de tabagismo que é um problema de saúde pública globalizado, que transcende as fronteiras dos países e cujo controle depende de medidas intersetoriais, fortalecendo e organizando a rede de atenção ao tabagista afim de que o usuário possa iniciar a abordagem e a farmacoterapia em tempo oportuno com a maior comodidade possível. Dessa forma, contribuindo com o abandono do vício ao tabaco, sendo de grande relevância na vida dos tabagistas e seus familiares, fumantes passivos, consequentemente diminuindo as chances de morbidades e mortalidade relacionadas ao consumo da nicotina proporcionando uma melhor qualidade de vida a todos os envolvidos.

\section{REFERÉNCIAS}

Araújo RB, Oliveira MS, Moraes JFD, Pedroso RS, Port F, de Castro MGT. Validação da versão brasileira do Questionnaire of Smoking Urges-Brief. Rev. psiquiatr. clín. [Internet] 2007;34(4) [acesso em 17 ago 2017]. Disponível: http://dx.doi.org/10.1590/S0101-60832007000400002.

Almeida, Aline Farias de; MUSSI, Fernanda Carneiro. Tabagismo: conhecimentos, atitudes, hábitos e grau de dependência de jovens fumantes em Salvador. 2006. [acesso em: 16 de nov 2018]. Disponível em: http://www.scielo.br/pdf/reeusp/v40n4/v40n4a01.pdf.

Boeira, Sergio Luiz. Indústria de tabaco e cidadania: confronto entre redes organizacionais. VOL. 46. №3. São José - SC, 2006. [acesso em: 14 de nov 2018]. Disponível em: http://www.scielo.br/pdf/rae/v46n3/v46n3a04.pdf.

Brasil. Ministério da Saúde. Cadernos da Atenção Básica, № 40. Estratégia para o cuidado da pessoa com doença crônica: o cuidado da pessoa tabagista, Brasília DF. 
2015. P.145. [acesso em: 21 de fev. 2018] Disponível em: http://189.28.128.100/dab/docs/portaldab/publicacoes/caderno_40.pdf.

Brasil - População - História. 2. Brasil - Colonização. 3. Brasil - População estrangeira. 4. Brasil - Migração - História. 5. Migração - Estatística. 6. Índios da América do Sul Brasil. 7. Negros - Brasil. I. IBGE. Centro de Documentação e Disseminação de Informações. Divisão de Biblioteca e Acervos Especiais CDU 314.148(81) RJ/2007-07. [acesso em: 12 de nov. 2018] Disponível em:https://biblioteca.ibge.gov.br/visualizacao/livros/liv6687.pdf

Ministério da Saúde. Número de fumantes no País diminuiu nos últimos 25 anos. Publicado por Portal Brasil: 17/04/2017. [acesso em: 10 de dez 2018]. Disponível em: http://www.brasil.gov.br/saude/2017/04/Numero-de-fumantes-no-pais-diminuiunos-ultimos-25-anos.

Ministério da Saúde. Instituto Nacional de Câncer - INCA. Coordenação de Prevenção e Vigilância (CONPREV). Abordagem e Tratamento do Fumante, Rio de Janeiro, 2001. [acesso em: 14 de nov 2018]. Disponível em: http://www.saude.sp.gov.br/resources/ses/perfil/profissional-dasaude/homepage//tratamento_fumo_consenso.pdf.

Ministério da Saúde. Cigarro mata mais de 5 milhões de pessoas, segundo OMS. Última modificação: 23/12/2017 11h30. [acesso em: 17 de out 2018]. Disponível em: http://www.brasil.gov.br/noticias/saude/2014/08/cigarro-mata-mais-de-5milhoesde-pessoas-segundo-oms.

Ministério da Saúde. Instituto Nacional de câncer- INCA, 2007. Doenças associadas ao tabagismo. [acesso em: 11 de out 2018]. Disponível em: http://www.inca.gov.br/conteudo_view.asp?id=2588.

Caram, L.M.O. et al. Perfil de fumantes atendidos em serviço público para tratamento do tabagismo. Botucatu (SP) Brasil, 17 de Mar. 2009. [acesso em: 11 fev de 2018]. Disponível em: http://www.scielo.br/pdf/jbpneu/v35n10/v35n10a06.pdf.

Echer, Isabel Cristina, et al. Tabagismo em uma Escola de Enfermagem do Sul do Brasil. Enferm. Florianópolis, 2011. [acesso em: 18 de nov 2018]. Disponível em:

Figueiredo, Valeska Carvalho. et al. ERICA: prevalência de tabagismo em adolescentes brasileiros. Revista Saúde Pública. Brasil, Rio de Janeiro, RJ, 2016. [acesso em: 20 de fev 2018]. Disponível em: http://www.scielo.br/pdf/rsp/v50s1/pt_0034-8910-rsp-S0151887872016050006741.pdf.

Halty, Luis Suárez, et al. Análise da utilização do Questionário de Tolerância de Fagerström (QTF) como instrumento de medida da dependência nicotínica. [acesso em: 16 de nov 2018]. Disponível em: http://www.scielo.br/pdf/jpneu/v28n4/12962.pdf.

Luppi, Claudia Helena Brozato; Alves, Maria Virgínia Faria Faddul Santos Alana Arantes. Programa de Cessação ao Tabagismo: Perfil e resultados. Rev. Ciênc. Ext. v.2, n.2, 
p.13, 2008. [acesso em: 13 de nov 2018]. Disponível em: http://ojs.unesp.br/index.php/revista_proex/article/viewFile/196/109.

Medronho, R.A. Epidemiologia. São Paulo: Atheneu; 2002.

Müller, Erildo V. et al. Fatores associados ao tabagismo em usuários da estratégia saúde da família. Revista Cogitare Enferm. (22)4: e50600, 2017. Brasil. Ponta Grossa, PR, 2017. [acesso em: 20 de dez 2018].Disponível em: DOI: http://dx.doi.org/10.5380/ce.v22i4.50600

Nunes, Sandra Odebrecht Vargas; Castro, Márcia Regina Pizzo; Castro, Mario Sérgio Azenha de. Tabagismo, Morbidades e danos à saúde. EDUEL, Londrina, 2011. [acesso em: 14 de ago 2018]. Disponível em: http://books.scielo.org/id/sj9xk/pdf/nunes9788572166751-01.pdf.

Rosemberg, Jose; rosemberg, Ana Margarida Aruda; MORAES, Marco Antônio de. Nicotina Droga Universal, Monografia. Produção Independente. São Paulo: 2004.p. 33. [acesso em: 16 de nov 2018]. Disponível em: https://pt.scribd.com/document/73685616/Nicotina-Droga-Universal-Livro.

Rossaneis. Mariana Ângela; MACHADO, Regina Célia Bueno Rezendes. Cessação do tabagismo em pacientes assistidos em um ambulatório de tratamento de dependência do tabaco. Cienc. cuid. saude. v10i2.15688. Rolândia, Paraná, 2011. [acesso em: 05 de nov 2018]. Disponível em: file:///C:/Users/Terezinha/Downloads/15688-64087-1PB\%20(1).pdf.

Schmidt, Maria linês, et al. Doenças crônicas não transmissíveis no Brasil: carga e desafios atuais. Saúde do Brasil 4. Porto Alegre, RS, Brasil, 2011. [acesso em: 16 de nov 2018]. Disponível em: http://www.idec.org.br/pdf/schmidtetal_lancet2011.pdf.

Zaitune, Maria Paula de Araujo, et al. Fatores associados ao tabagismo em idosos: Inquérito de Saúde no Estado de São Paulo (ISA-SP). Cad. Saúde Pública, Rio de Janeiro, 2012. [acesso em: 05 de nov 2018]. Disponível em: https://www.scielosp.org/article/csp/2012.v28n3/583-596/

Zancan, N. et al. Intervenções psicológicas em grupos de controle de tabagismo: relato de experiência. Revista de Psicologia da IMED, v.3, n.2, p. 534-544, 2011. [acesso em: 08 de nov 2018]. Disponível em: https://seer.imed.edu.br/index.php/revistapsico/article/view/132/109. 